\title{
Review Article \\ The State of Charge Estimating Methods for Battery: A Review
}

\author{
Wen-Yeau Chang \\ Department of Electrical Engineering, St. John's University, 499, Sec. 4, Tam King Road, Tamsui District, New Taipei City 25135, Taiwan
}

Correspondence should be addressed to Wen-Yeau Chang; changwy@mail.sju.edu.tw

Received 12 May 2013; Accepted 5 July 2013

Academic Editors: M. Brünig and E. Di Nardo

Copyright (C) 2013 Wen-Yeau Chang. This is an open access article distributed under the Creative Commons Attribution License, which permits unrestricted use, distribution, and reproduction in any medium, provided the original work is properly cited.

\begin{abstract}
An overview of new and current developments in state of charge (SOC) estimating methods for battery is given where the focus lies upon mathematical principles and practical implementations. As the battery SOC is an important parameter, which reflects the battery performance, so accurate estimation of SOC cannot only protect battery, prevent overcharge or discharge, and improve the battery life, but also let the application make rationally control strategies to achieve the purpose of saving energy. This paper gives a literature survey on the categories and mathematical methods of SOC estimation. Based on the assessment of SOC estimation methods, the future development direction of SOC estimation is proposed.
\end{abstract}

\section{Introduction}

Rising crude oil prices and worldwide awareness of environmental issues have resulted in increased development of energy storage systems. The battery is one of the most attractive energy storage systems because of its high efficiency and low pollution [1]. There are several kinds of batteries currently being used in industry: lead-acid battery, Ni-MH battery, $\mathrm{Ni}$-Cd battery, and Li-ion battery. The battery has the advantages of high working cell voltage, low pollution, low self-discharge rate, and high power density. Batteries are used commonly for portable utilities, hybrid electric vehicles, and industrial applications [2].

SOC estimation is a fundamental challenge for battery use. The SOC of a battery, which is used to describe its remaining capacity, is a very important parameter for a control strategy [3]. As the SOC is an important parameter, which reflects the battery performance, so accurate estimation of the SOC can not only protect battery, prevent overdischarge, and improve the battery life but also allow the application to make rational control strategies to save energy [4]. However, a battery is a chemical energy storage source, and this chemical energy cannot be directly accessed. This issue makes the estimation of the SOC of a battery difficult [5]. Accurate estimation of the SOC remains very complex and is difficult to implement, because battery models are limited and there are parametric uncertainties [6]. Many examples of poor accuracy and reliability of the estimation of the SOC are found in practice [7].

This paper presents a detailed review on existing mathematical methods used in SOC estimation and further identifies possible developments in the future.

\section{Definition and Classification of SOC Estimation}

The SOC is one of the most important parameters for batteries, but its definition presents many different issues [5]. In general, the SOC of a battery is defined as the ratio of its current capacity $(Q(t))$ to the nominal capacity $\left(Q_{n}\right)$. The nominal capacity is given by the manufacturer and represents the maximum amount of charge that can be stored in the battery. The SOC can be defined as follows:

$$
\operatorname{SOC}(t)=\frac{Q(t)}{Q_{n}}
$$

The various mathematical methods of estimation are classified according to methodology. The classification of these SOC estimation methods is different in the various literatures. However, some literatures $[5,7]$ allow a division into the following four categories. 
(i) Direct measurement: this method uses physical battery properties, such as the voltage and impedance of the battery.

(ii) Book-keeping estimation: this method uses discharging current as the input and integrates the discharging current over time to calculate the SOC.

(iii) Adaptive systems: the adaptive systems are selfdesigning and can automatically adjust the SOC for different discharging conditions. Various new adaptive systems for SOC estimation have been developed.

(iv) Hybrid methods: the hybrid models benefit from the advantages of each SOC estimation method and allow a globally optimal estimation performance. The literature shows that the hybrid methods generally produce good estimation of SOC, compared to individual methods.

Table 1 presents the specific SOC estimation methods in view of the methodology. The applications of specific SOC estimation methods in battery management system (BMS) are consequentially different.

\section{Overview of SOC Estimating Mathematical Methods}

3.1. Direct Measurement. Direct measurement methods refer to some physical battery properties such as the terminal voltage and impedance. Many different direct methods have been employed: open circuit voltage method, terminal voltage method, impedance measurement method, and impedance spectroscopy method.

3.1.1. Open Circuit Voltage Method. There is approximately a linear relationship between the SOC of the lead-acid battery and its open circuit voltage (OCV) given by

$$
V_{\mathrm{OC}}(t)=a_{1} \times \mathrm{SOC}(t)+a_{0},
$$

where $\operatorname{SOC}(t)$ is the SOC of the battery at $t, a_{0}$ is the battery terminal voltage when SOC $=0 \%$, and $a_{1}$ is obtained from knowing the value of $a_{0}$ and $V_{\mathrm{OC}}(t)$ at SOC $=100 \%$. By (2), the estimation of the SOC is equivalent to the estimation of its OCV [8]. The OCV method based on the OCV of batteries is proportional to the SOC when they are disconnected from the loads for a period longer than two hours. However, such a long disconnection time may be too long to be implemented for battery [9].

Unlike the lead-acid battery, the Li-ion battery does not have a linear relationship between the OCV and SOC [10]. A typical relationship of Li-ion battery between SOC and OCV is shown in Figure 1 [11]. The OCV relationship with SOC was determined from applying a pulse load on the Li-ion battery, then allowing the battery to reach equilibrium [12].

The relationship between the OCV and SOC cannot be exactly the same for all batteries. Because the conventional OCV-SOC differs among batteries, there is a problem in that the relationship of the OCV-SOC should be measured to estimate accurately the SOC. Lee et al. [13] proposed a
TABLE 1: Classification of SOC estimating mathematical methods.

\begin{tabular}{|c|c|}
\hline Categories & Mathematical methods \\
\hline \multirow{4}{*}{ Direct measurement } & (i) Open circuit voltage method \\
\hline & (ii) Terminal voltage method \\
\hline & (iii) Impedance method \\
\hline & $\begin{array}{l}\text { (iv) Impedance spectroscopy } \\
\text { method }\end{array}$ \\
\hline \multirow{2}{*}{ Book-keeping estimation } & (i) Coulomb counting method \\
\hline & $\begin{array}{l}\text { (ii) Modified Coulomb counting } \\
\text { method }\end{array}$ \\
\hline \multirow{5}{*}{ Adaptive systems } & (i) BP neural network \\
\hline & (ii) RBF neural network \\
\hline & (iii) Support vector machine \\
\hline & (iv) Fuzzy neural network \\
\hline & (v) Kalman filter \\
\hline \multirow{3}{*}{ Hybrid methods } & $\begin{array}{l}\text { (i) Coulomb counting and EMF } \\
\text { combination }\end{array}$ \\
\hline & $\begin{array}{l}\text { (ii) Coulomb counting and Kalman } \\
\text { filter combination }\end{array}$ \\
\hline & $\begin{array}{l}\text { (iii) Per-unit system and EKF } \\
\text { combination }\end{array}$ \\
\hline
\end{tabular}

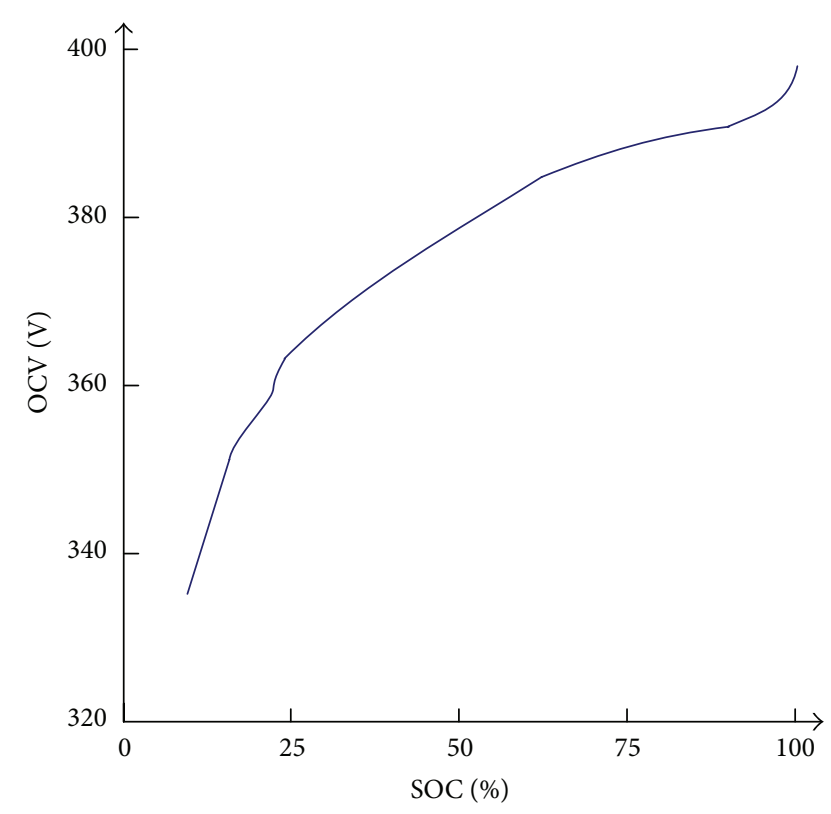

FIgURE 1: Typical relationship between SOC and OCV [11].

modified OCV-SOC relationship based on the conventional OCV-SOC. The SOC and the capacity of a lithium-ion battery are estimated using the dual extended Kalman filter with the proposed method.

3.1.2. Terminal Voltage Method. The terminal voltage method is based on the terminal voltage drops because of the internal impedances when the battery is discharging, so the electromotive force (EMF) of battery is proportional to the terminal voltage. Since the EMF of battery is approximately 
linear proportional to the SOC, the terminal voltage of battery is also approximately linear proportional to the SOC. The terminal voltage method has been employed at different discharge currents and temperatures [14]. But at the end of battery discharge, the estimated error of terminal voltage method is large, because the terminal voltage of battery suddenly drops at the end of discharge [15].

3.1.3. Impedance Method. Among the techniques which have been employed, impedance measurements provide knowledge of several parameters, the magnitudes of which may depend on the SOC of the battery. Although the impedance parameters and their variations with SOC are not unique for all battery systems, it appears to be imperative to perform a wide range of impedance experiments for identification and use of impedance parameters for estimating the SOC of a given battery $[16,17]$.

3.1.4. Impedance Spectroscopy Method. The impedance spectroscopy method measures battery impedances over a wide range of ac frequencies at different charge and discharge currents. The values of the model impedances are found by least-squares fitting to measured impedance values. SOC may be indirectly inferred by measuring present battery impedances and correlating them with known impedances at various SOC levels $[18,19]$.

3.2. Book-Keeping Estimation. Book-keeping estimation method uses battery discharging current data as input. This method permits to include some internal battery effects as self-discharge, capacity-loss, and discharging efficiency. Two kinds of book-keeping estimation methods have been employed: Coulomb counting method and modified Coulomb counting method.

3.2.1. Coulomb Counting Method. The Coulomb counting method measures the discharging current of a battery and integrates the discharging current over time in order to estimate SOC [20]. Coulomb counting method is done to estimate the $\operatorname{SOC}(t)$, which is estimated from the discharging current, $I(t)$, and previously estimated SOC values, SOC $(t-$ 1). SOC is calculated by the following equation:

$$
\operatorname{SOC}(t)=\operatorname{SOC}(t-1)+\frac{I(t)}{Q_{n}} \Delta t .
$$

But there are several factors that affect the accuracy of Coulomb counting method including temperature, battery history, discharge current, and cycle life [20].

3.2.2. Modified Coulomb Counting Method. To improve the Coulomb counting method, a new technique called modified Coulomb counting method is proposed. The modified Coulomb counting method uses the corrected current to improve the accuracy of estimation.

The corrected current is the function of discharging current. There is a quadratic relationship between the corrected current and discharging current of battery. By practice of experimental data, corrected current is calculated by the following form:

$$
I_{c}(t)=k_{2} I(t)^{2}+k_{1} I(t)+k_{0},
$$

where $k_{2}, k_{1}$ and $k_{0}$ are constant values obtained from the practice experimental data.

In modified Coulomb counting method, SOC is calculated by the following equation:

$$
\operatorname{SOC}(t)=\operatorname{SOC}(t-1)+\frac{I_{c}(t)}{Q_{n}} \Delta t .
$$

The experimental results show that the accuracy of the modified Coulomb counting method is superior to the conventional Coulomb counting method.

3.3. Adaptive Systems. Recently, with the development of artificial intelligence, various new adaptive systems for SOC estimation have been developed. The new developed methods include back propagation (BP) neural network, radial basis function (RBF) neural network, fuzzy logic methods, support vector machine, fuzzy neural network, and Kalman filter. The adaptive systems are self-designing ones that can be automatically adjusted in changing systems. As batteries have been affected by many chemical factors and have nonlinear SOC, adaptive systems offer good solution for SOC estimation [5].

3.3.1. BP Neural Network. BP neural network is the most popular type in artificial neural networks. The BP neural network is applied in SOC estimation due to their good ability of nonlinear mapping, self-organization, and self-learning [1]. As the problem defined, the relationship between the input and target is nonlinear and very complicated in SOC estimation [21]. The artificial neural network based SOC indicator predicts the current SOC using the recent history of voltage, current, and the ambient temperature of a battery [22].

The architecture of the SOC estimating BP neural network is shown in Figure 2. The architecture of BP neural network contains an input layer, an output layer, and a hidden layer. Input layer has 3 neurons for terminal voltage, discharge current, and temperature, hidden layer has $g$ neurons, and output layer has only one neuron for SOC [1].

The total input of a neuron in hidden layer is calculated by the following form:

$$
\operatorname{net}_{j}=\sum_{i=1}^{3} x_{i} v_{i j}+b_{j},
$$

where net $i_{j}$ is total input of the hidden layer neuron $j ; x_{i}$ is input to the hidden layer neuron $j$ from input layer neuron $i$; $v_{i j}$ is weight between the input layer neuron $i$ and hidden layer neuron $j ; b_{j}$ is bias of the hidden layer neuron $j$.

The activation function applied to neuron in hidden layer is the hyperbolic tangent function which is calculated by the following equation:

$$
h_{j}=f\left(\text { net } i_{j}\right)=\frac{1-e^{-2 \text { net } i_{j}}}{1+e^{-2 \text { net }} i_{j}} .
$$




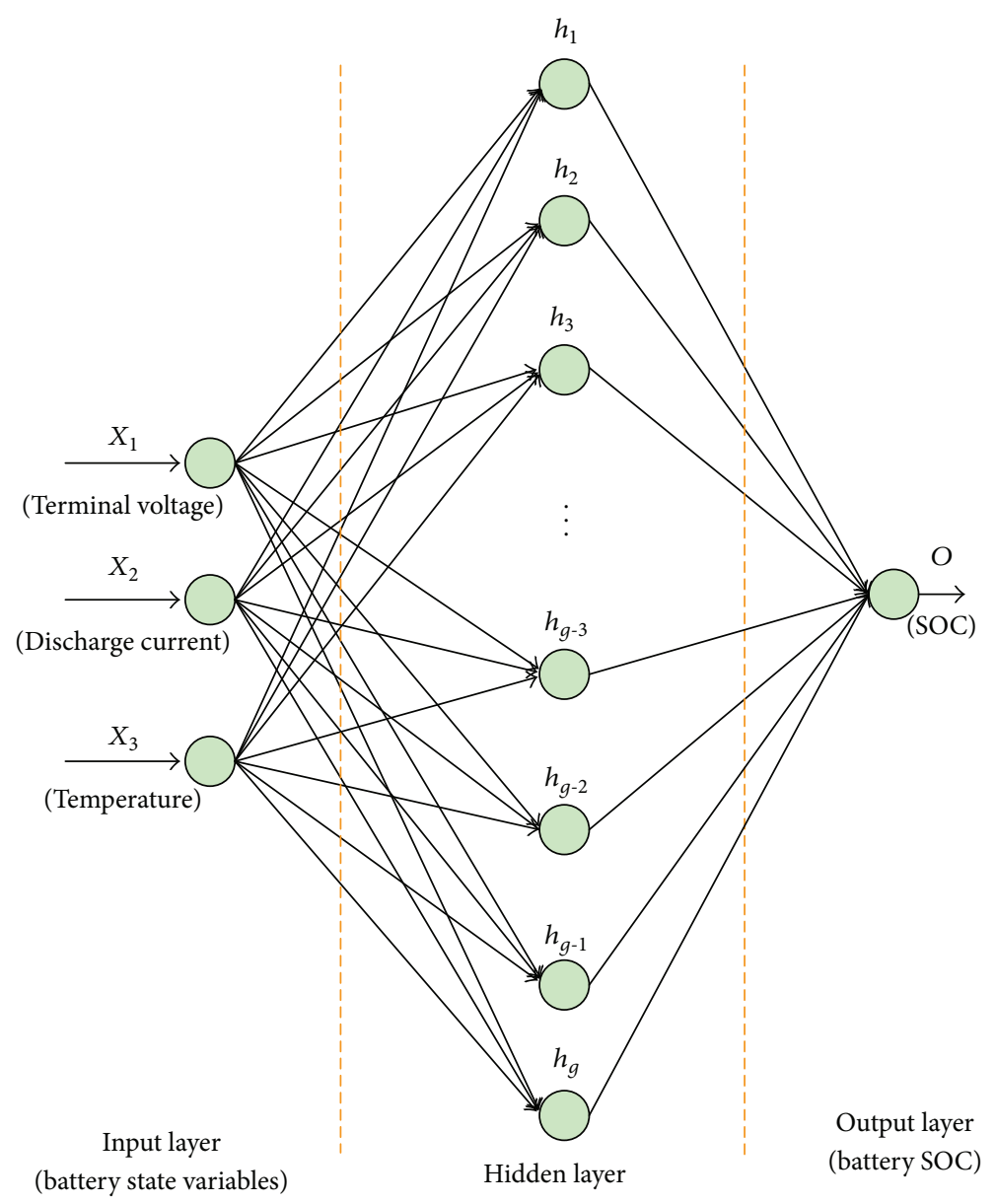

FIGURE 2: The architecture of the SOC estimating BP neural network [1]. by

The total input of the neuron $o$ in output layer is calculated

$$
\text { net } o=\sum_{i=1}^{g} h_{i} w_{i}+k,
$$

where net $o$ is total input of the output layer neuron $o$; $h_{i}$ is input to the output layer neuron from hidden layer neuron $i$; $w_{i}$ is weight between the hidden layer neuron $i$ and output layer neuron; $k$ is bias of the output layer neuron $o ; g$ is number of neurons in the hidden layer.

The activation function applied to neuron $o$ in output layer is the sigmoid function as the following equation:

$$
o=f(\text { net } o)=\frac{1}{1+e^{- \text {net } o}} .
$$

3.3.2. RBF Neural Network. The RBF neural network is a useful estimation methodology for systems with incomplete information. It can be used to analyze the relationships between one major (reference) sequence and the other comparative ones in a given set. The RBF neural network has been used in SOC estimation. The method was tested with data which was from battery experiments. Results show that the operation speed and estimation accuracy of estimating model can meet the demands in practice, and the model has certain value of application $[23,24]$.

In [1], the RBF neural network SOC estimation method uses the input data of the terminal voltage, discharging current, and temperature of battery to estimate the SOC for $\mathrm{LiFePO}_{4}$ battery under different discharging conditions. The experimental data are found to be in close agreement.

3.3.3. Fuzzy Logic Method. Fuzzy logic method provides a powerful means of modeling nonlinear and complex systems. In [25], a practical method of estimating SOC of battery system has been developed and tested for several systems. The method involves the use of fuzzy logic models to analyze data obtained by impedance spectroscopy and/or Coulomb counting methods. In [26], a fuzzy logic-based SOC estimation method has been developed for lithium-ion batteries for potential use in portable defibrillators. The ac impedance and voltage recovery measurements have been made which are used as the input parameters for the fuzzy logic model.

Singh et al. [27] presented an estimation system which can select features in data base to develop fuzzy logic models 
for both available capacity and SOC estimation, simply by measuring the impedance at three frequencies. In [28], the SOC is estimated by an improved Coulomb metric method, and the time-dependent variation is compensated by using a learning system. The learning system tunes the Coulomb metric method in such a way that the estimation process remains error free from the time-dependent variation. The proposed learning system uses the fuzzy logic models, which is not used for estimation of SOC but performs as a component of learning system.

3.3.4. Support Vector Machine. The support vector machine (SVM) has been applied for classification in various domains of pattern recognition. The SVM has also been applied for regression problem, even the regression problem inherently more difficult than classification problem. The SVM used as a nonlinear estimation system is more robust than a leastsquares estimation system because it is insensitive to small changes [29].

Hansen and Wang [29] investigated the application of a SVM to estimate the SOC of lithium-ion battery. The SVM based estimator not only removes the drawbacks of the Coulomb counting SOC estimator but also produces accurate SOC estimates.

3.3.5. Fuzzy Neural Network. Fuzzy neural network (FNN) has been used in many applications, especially in identification of unknown systems. In nonlinear system identification, FNN can effectively fit the nonlinear system by calculating the optimized coefficients of the learning mechanism [30].

Lee et al. [31] investigated a soft computing technique for estimating battery SOC of individual batteries in a battery string. The soft computing approach uses a fusion of an FNN with B-spline membership functions and a reduced-form genetic algorithm.

3.3.6. Kalman Filter. Using real-time measurement road data to estimate the SOC of battery would normally be difficult or expensive to measure. In [32], application of the Kalman filter method is shown to provide verifiable estimations of SOC for the battery via the real-time state estimation.

Yatsui and Bai [33] presented a Kalman filter based SOC estimation method for lithium-ion batteries. Experimental results validate the effectiveness of Kalman filter during the online application. Barbarisi et al. [34] presented an extended Kalman filter (EKF) to estimate the concentrations of the main chemical species which are averaged on the thickness of the active material in order to obtain the SOC of the battery, by using the terminal current and voltage measurements.

Based on unscented Kalman filter (UKF) theory and a comprehensive battery model, a novel SOC estimation method is proposed in [35]. The results show that UKF method is superior to extended Kalman filter method in SOC estimation for battery. Sun et al. [36] presented an adaptive UKF method to estimate SOC of a lithium-ion battery for battery electric vehicles. The adaptive adjustment of the noise covariance in the SOC estimation process is implemented by an idea of covariance matching in the UKF context.
3.4. Hybrid Methods. The object of hybrid models is to benefit from the advantages of each method and obtain a globally optimal estimating performance. Since the information contained in the individual estimating method is limited, hybrid method can maximize the available information, integrate individual model information, and make the best use of the advantages of multiple estimating methods thus improving the estimation accuracy. The literatures show that the hybrid methods generally produce good SOC estimating results compared to individual methods [37-39]. The hybrid methods combine different approaches such as direct measurement method and book-keeping estimation method.

3.4.1. Coulomb Counting and EMF Combination. A new SOC estimation method that combines direct measurement method with the battery EMF measurement during the equilibrium state and book-keeping estimation with Coulomb counting method during the discharge state has been developed and implemented in a real-time estimation system [37].

Any battery will lose capacity during cycling. In order to calculate SOC and remaining run-time (RRT) accurately and to improve the SOC estimation system capability to cope with the aging effect, a simple Qmax adaptation algorithm is introduced. In this algorithm the stable conditions of the charge state are exploited in order to adapt Qmax with the aging effect.

This paper has proved that the Qmax adaptation algorithm can improve the SOC and RRT estimation accuracy even for a fresh battery. Since a battery loses capacity during cycling, it is concluded that the Qmax adaptation algorithm will increase substantially the SOC and the RRT estimation accuracy.

3.4.2. Coulomb Counting and Kalman Filter Combination. Wang et al. [38] proposed a new SOC estimation method, denoted as "KalmanAh method," which uses the Kalman filter method to correct for the initial value used in the Coulomb counting method. In KalmanAh method, the Kalman filter method is used to make the approximate initial value converge to its real value. Then the Coulomb counting method is applied to estimate the SOC for the long working time. The SOC estimation error is $2.5 \%$ when compared with the real SOC obtained from a discharge test. This compares favorably with an estimation error of $11.4 \%$ when using Coulomb counting method.

3.4.3. Per-Unit System and EKF Combination. Kim and Cho [39] described the application of an EKF combined with a per-unit (PU) system to the identification of suitable battery model parameters for the high accuracy SOC estimation of a lithium-ion degraded battery. To apply the battery model parameters varied by the aging effect, based on the PU system, the absolute values of the parameters in the equivalent circuit model in addition to the terminal voltage and current are converted into dimensionless values relative to a set of base value. The converted values are applied to dynamic and measurement models in the EKF algorithm. 


\section{The Future of SOC Estimation}

Since the energy storage systems have been highlighted in portable electronics and hybrid electric vehicle applications, the estimate accuracy of SOC becomes increasingly important. In recent years, many scholars have done a lot of research on SOC estimation. The estimate accuracy has improved constantly, and it can be expected that intense research and development efforts are already on track. In order to further improve SOC estimates, combined with some literatures, anticipated improvements for the further research include the following areas.

(i) Do further research on the hybrid methods, such as combining direct measurement method and bookkeeping estimation method to achieve good results in online SOC estimation.

(ii) The existing estimation method should be put into use in various kinds of batteries. Do further research on the practical universal application of the methods.

(iii) Deepen further research on improve the SOC estimation system capability to cope with the aging effect of battery.

(iv) Study on more novel artificial intelligence methods and improving their training algorithms to achieve the estimate accuracy of SOC. In addition, new methods on complex terrain are the focus of future research.

(v) To further improve the estimating performance of the neural network method, the optimal search methods for the optimal number of neurons in hidden layer need to be investigated and integrated in the neural network method.

(vi) Do further research on the adaptive parameter estimation. The models have the ability to automatically adapt to various kinds of batteries, various discharging conditions, and the different aged batteries.

(vii) Establish the more accurate evaluation system and the standard for measurement of performance of SOC estimation method.

\section{Conclusions}

This paper presented a review on estimating of battery SOC under different discharging conditions. Four categories of estimating mathematical methods, which have their own characteristics, were discussed. Papers were selected to emphasize the diversity of estimating mathematical methods. Some of these methods have good performances at fixed discharging current condition, while others perform better in varied discharging current condition. It is difficult to evaluate the performance of various methods, as the existing applications were in different discharging condition and different size of battery. The developments of various SOC estimate methods are expected to be valuable in battery applications such as BMS in hybrid electric vehicles. Based on the development history of SOC estimation, the future development directions of SOC estimating are proposed in the end.

\section{Acknowledgment}

The author would like to express his gratitude to the National Science Council of ROC for the financial support under Grant no. NSC 101-2221-E-129-005.

\section{References}

[1] W. Y. Chang, "State of charge estimation for $\mathrm{LiFePO}_{4}$ battery using artificial neural network," International Review of Electrical Engineering, vol. 7, no. 5, pp. 5874-5800, 2012.

[2] Z. H. Rao, S. F. Wang, and G. Q. Zhang, "Simulation and experiment of thermal energy management with phase change material for ageing $\mathrm{LiFePO}_{4}$ power battery," Energy Conversion and Management, vol. 52, no. 12, pp. 3408-3414, 2011.

[3] H. W. He, R. Xiong, and H. Q. Guo, "Online estimation of model parameters and state-of-charge of $\mathrm{LiFePO}_{4}$ batteries in electric vehicles," Applied Energy, vol. 89, no. 1, pp. 413-420, 2012.

[4] Z. H. Cai, G. F. Liu, and J. Luo, "Research state of charge estimation tactics of nickel-hydrogen battery," in Proceedings of the International Symposium on Intelligence Information Processing and Trusted Computing (IPTC '10), pp. 184-187, Huanggang, China, October 2010.

[5] N. Watrin, B. Blunier, and A. Miraoui, "Review of adaptive systems for lithium batteries state-of-charge and state-of-health estimation," in Proceedings of IEEE Transportation Electrification Conference and Expo, pp. 1-6, Dearborn, Mich, USA, June 2012.

[6] A. A. A. Elgammal and A. M. Sharaf, "Self-regulating particle swarm optimised controller for (photovoltaic-fuel cell) battery charging of hybrid electric vehicles," IET Electrical Systems in Transportation, vol. 2, no. 2, pp. 77-89, 2012.

[7] V. Prajapati, H. Hess, E. J. William et al., "A literature review of state of-charge estimation techniques applicable to lithium poly-carbon monoflouride (LI/CFx) battery," in Proceedings of the India International Conference on Power Electronics (IICPE '10), pp. 1-8, New Delhi, India, January 2011.

[8] J. Chiasson and B. Vairamohan, "Estimating the state of charge of a battery," IEEE Transactions on Control Systems Technology, vol. 13, no. 3, pp. 465-470, 2005.

[9] K. S. Ng, C. S. Moo, Y. P. Chen, and Y. C. Hsieh, "State-of-charge estimation for lead-acid batteries based on dynamic opencircuit voltage," in Proceedings of the 2nd IEEE International Power and Energy Conference (PECon '08), pp. 972-976, Johor Bahru, Malaysia, December 2008.

[10] M. Coleman, C. K. Lee, C. Zhu, and W. G. Hurley, "Stateof-charge determination from EMF voltage estimation: using impedance, terminal voltage, and current for lead-acid and lithium-ion batteries," IEEE Transactions on Industrial Electronics, vol. 54, no. 5, pp. 2550-2557, 2007.

[11] T. Dong, J. Li, F. Zhao et al., "Analysis on the influence of measurement error on state of charge estimation of $\mathrm{LiFePO}_{4}$ power battery," in Proceedings of the International Conference on Materials for Renewable Energy and Environment (ICMREE '11), pp. 644-649, Shanghai, China, May 2011.

[12] S. Abu-Sharkh and D. Doerffel, "Rapid test and non-linear model characterisation of solid-state lithium-ion batteries," Journal of Power Sources, vol. 130, no. 1-2, pp. 266-274, 2004. 
[13] S. Lee, J. Kim, J. Lee, and B. H. Cho, "State-of-charge and capacity estimation of lithium-ion battery using a new opencircuit voltage versus state-of-charge," Journal of Power Sources, vol. 185, no. 2, pp. 1367-1373, 2008.

[14] A. H. Anbuky and P. E. Pascoe, "VRLA battery state-ofcharge estimation in telecommunication power systems," IEEE Transactions on Industrial Electronics, vol. 47, no. 3, pp. 565-573, 2000.

[15] S. Sato and A. Kawamura, "A new estimation method of state of charge using terminal voltage and internal resistance for lead acid battery," in Proceedings of the Power Conversion Conference, pp. 565-570, Osaka, Japan, April 2002.

[16] S. Rodrigues, N. Munichandraiah, and A. K. Shukla, "A review of state-of-charge indication of batteries by means of A.C. impedance measurements," Journal of Power Sources, vol. 87, no. 1-2, pp. 12-20, 2000.

[17] F. Huet, "A review of impedance measurements for determination of the state-of-charge or state-of-health of secondary batteries," Journal of Power Sources, vol. 70, no. 1, pp. 59-69, 1998.

[18] R. Li, J. F. Wu, H. Y. Wang, and G. C. Li, "Prediction of state of charge of lithium-ion rechargeable battery with electrochemical impedance spectroscopy theory," in Proceedings of the 5th IEEE Conference on Industrial Electronics and Applications (ICIEA '10), pp. 684-688, Taichung, Taiwan, June 2010.

[19] K. Bundy, M. Karlsson, G. Lindbergh, and A. Lundqvist, "An electrochemical impedance spectroscopy method for prediction of the state of charge of a nickel-metal hydride battery at open circuit and during discharge," Journal of Power Sources, vol. 72 , no. 2, pp. 118-125, 1998.

[20] K. S. Ng, C. S. Moo, Y. P. Chen, and Y. C. Hsieh, "Enhanced Coulomb counting method for estimating state-of-charge and state-of-health of lithium-ion batteries," Applied Energy, vol. 86, no. 9, pp. 1506-1511, 2009.

[21] T. Weigert, Q. Tian, and K. Lian, "State-of-charge prediction of batteries and battery-supercapacitor hybrids using artificial neural networks," Journal of Power Sources, vol. 196, no. 8, pp. 4061-4066, 2011.

[22] O. Linda, E. J. William, M. Huff et al., "Intelligent neural network implementation for SOCI development of $\mathrm{Li} / \mathrm{CFx}$ batteries," in Proceedings of the 2nd International Symposium on Resilient Control Systems (ISRCS '09), pp. 57-62, Idaho Falls, Idaho, USA, August 2009.

[23] H. Guo, J. Jiang, and Z. Wang, "Estimating the state of charge for Ni-MH battery in HEV by RBF neural network," in Proceedings of the International Workshop on Intelligent Systems and Applications (ISA '09), pp. 1-4, Wuhan, China, May 2009.

[24] W. He, D. Huang, and D. Feng, "The prediction of SOC of lithium batteries and varied pulse charge," in Proceedings of IEEE International Conference on Mechatronics and Automation (ICMA '09), pp. 1578-1582, Changchun, China, August 2009.

[25] A. J. Salkind, C. Fennie, P. Singh, T. Atwater, and D. E. Reisner, "Determination of state-of-charge and state-of-health of batteries by fuzzy logic methodology," Journal of Power Sources, vol. 80, no. 1-2, pp. 293-300, 1999.

[26] P. Singh, R. Vinjamuri, X. Wang, and D. Reisner, "Design and implementation of a fuzzy logic-based state-of-charge meter for Li-ion batteries used in portable defibrillators," Journal of Power Sources, vol. 162, no. 2, pp. 829-836, 2006.

[27] P. Singh, C. Fennie Jr., and D. Reisner, "Fuzzy logic modelling of state-of-charge and available capacity of nickel/metal hydride batteries," Journal of Power Sources, vol. 136, no. 2, pp. 322-333, 2004.

[28] S. Malkhandi, "Fuzzy logic-based learning system and estimation of state-of-charge of lead-acid battery," Engineering Applications of Artificial Intelligence, vol. 19, no. 5, pp. 479-485, 2006.

[29] T. Hansen and C. J. Wang, "Support vector based battery state of charge estimator," Journal of Power Sources, vol. 141, no. 2, pp. 351-358, 2005.

[30] I. H. Li, W. Y. Wang, S. F. Su, and Y. S. Lee, "A merged fuzzy neural network and its applications in battery state-of-charge estimation," IEEE Transactions on Energy Conversion, vol. 22, no. 3, pp. 697-708, 2007.

[31] Y. S. Lee, W. Y. Wang, and T. Y. Kuo, "Soft computing for battery state-of-charge (BSOC) estimation in battery string systems," IEEE Transactions on Industrial Electronics, vol. 55, no. 1, pp. 229-239, 2008.

[32] L. Xu, J. P. Wang, and Q. S. Chen, "Kalman filtering state of charge estimation for battery management system based on a stochastic fuzzy neural network battery model," Energy Conversion and Management, vol. 53, no. 1, pp. 33-39, 2012.

[33] M. W. Yatsui and H. Bai, "Kalman filter based state-of-charge estimation for lithium-ion batteries in hybrid electric vehicles using pulse charging," in Proceedings of the 7th IEEE Vehicle Power and Propulsion Conference (VPPC '11), pp. 1-5, Chicago, Ill, USA, September 2011.

[34] O. Barbarisi, F. Vasca, and L. Glielmo, "State of charge Kalman filter estimator for automotive batteries," Control Engineering Practice, vol. 14, no. 3, pp. 267-275, 2006.

[35] J. Zhang and C. Xia, "State-of-charge estimation of valve regulated lead acid battery based on multi-state unscented Kalman filter," International Journal of Electrical Power \& Energy Systems, vol. 33, no. 3, pp. 472-476, 2011.

[36] F. Sun, X. Hu, Y. Zou, and S. Li, "Adaptive unscented Kalman filtering for state of charge estimation of a lithium-ion battery for electric vehicles," Energy, vol. 36, no. 5, pp. 3531-3540, 2011.

[37] V. Pop, H. J. Bergveld, P. H. L. Notten, J. H. G. Op het Veld, and P. P. L. Regtien, "Accuracy analysis of the state-of-charge and remaining run-time determination for lithium-ion batteries," Measurement, vol. 42, no. 8, pp. 1131-1138, 2009.

[38] J. Wang, B. Cao, Q. Chen, and F. Wang, "Combined state of charge estimator for electric vehicle battery pack," Control Engineering Practice, vol. 15, no. 12, pp. 1569-1576, 2007.

[39] J. Kim and B. H. Cho, "State-of-charge estimation and stateof-health prediction of a Li-ion degraded battery based on an EKF combined with a per-unit system," IEEE Transactions on Vehicular Technology, vol. 60, no. 9, pp. 4249-4260, 2011. 


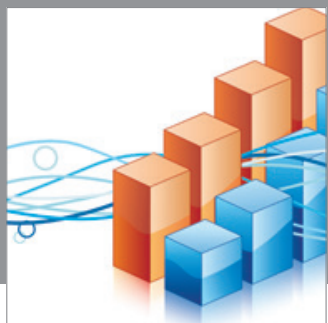

Advances in

Operations Research

mansans

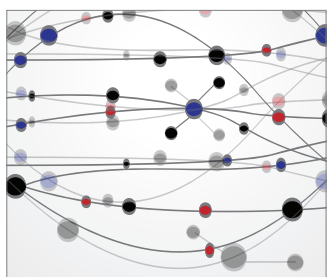

The Scientific World Journal
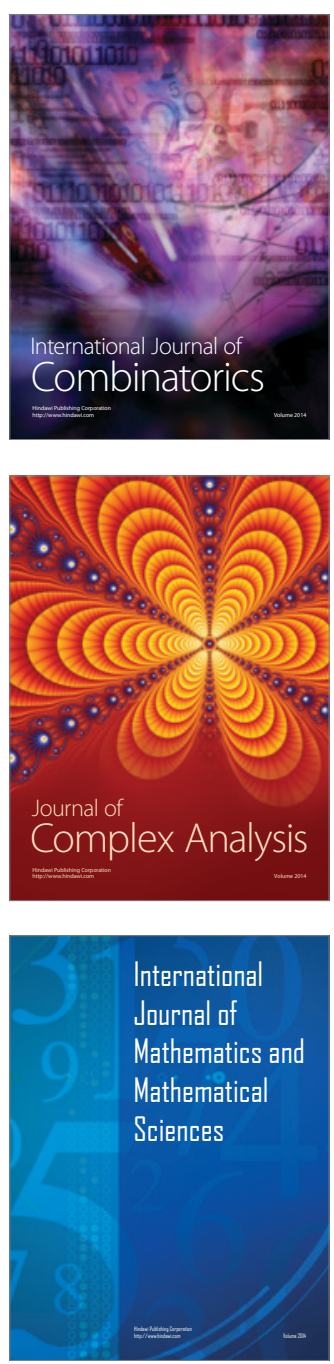
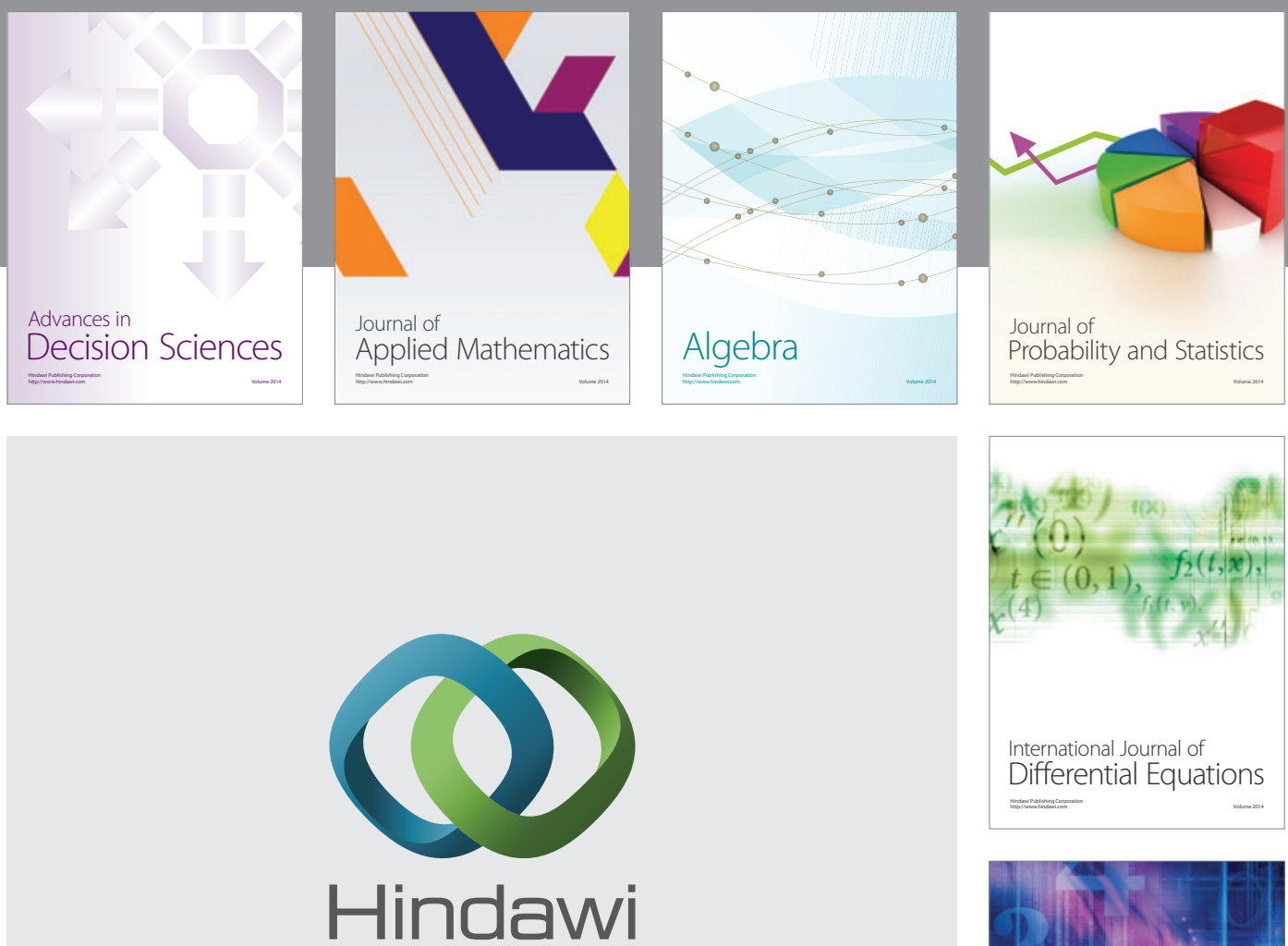

Submit your manuscripts at http://www.hindawi.com
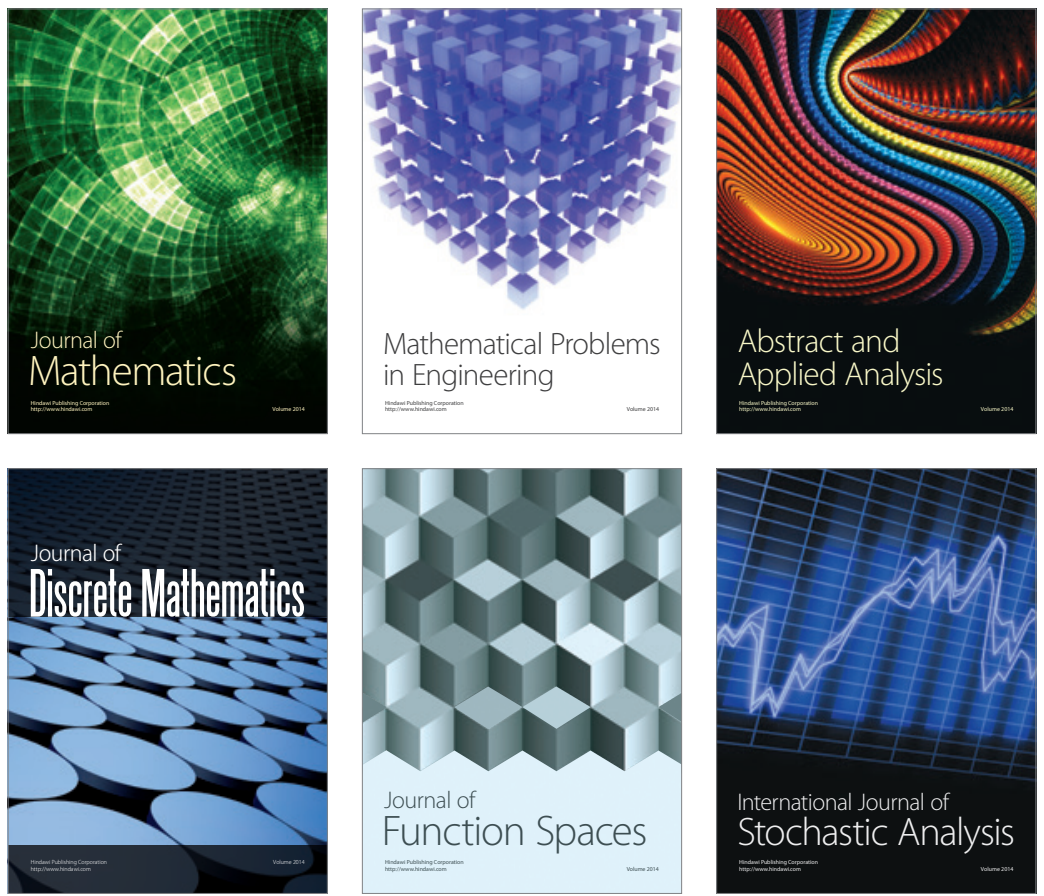

Journal of

Function Spaces

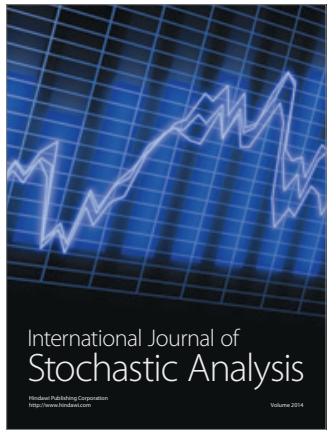

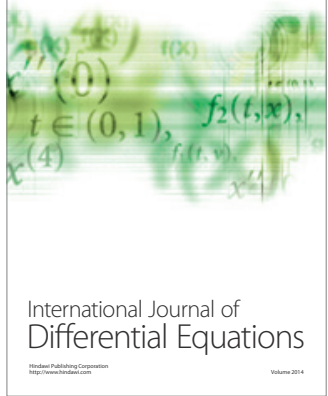
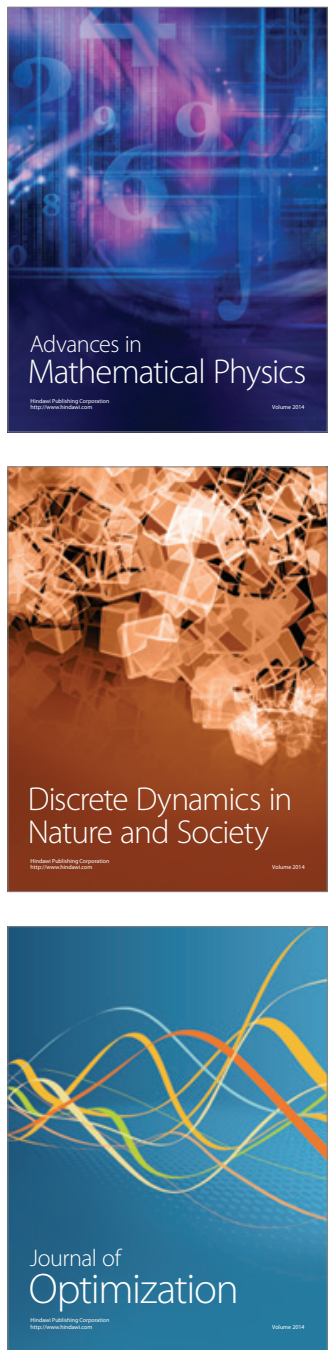